\title{
Hydrodynamic Modeling of Krypton and Xenon Propellant Performance in a Hall Thruster
}

\author{
John T. Yim, Michael Keidarł Jesse A. Linnellł; Alec D. Gallimore§, and Iain D. Boyd \\ University of Michigan, Ann Arbor, MI 48109, USA
}

\begin{abstract}
An axisymmetric hydrodynamic model of the plasma flow within a Hall thruster channel is used to model the differences between krypton and xenon propellants. Krypton offers a higher specific impulse and lower cost than xenon, but its overall efficiency is often lower. A greater understanding of the reasons behind this result would be helpful to allow future designs of Hall thrusters to utilize krypton as an efficient propellant. The model results are compared to experimental data obtained for the NASA-173Mv1 Hall thruster running at $500 \mathrm{~V}$ and $9.27 \mathrm{~A}$. The acceleration region for krypton is observed to be wider than that for xenon in both the model and experiment which is mainly due to the differences in the magnetic field profiles.
\end{abstract}

\section{Nomenclature}

\begin{tabular}{ll}
$B$ & Magnetic field $[\mathrm{T}]$ \\
$E$ & Electric field $[\mathrm{V} / \mathrm{m}]$ \\
$E_{\text {ion }}$ & Ionization energy $[\mathrm{eV}]$ \\
$I_{d}$ & Discharge current $[\mathrm{A}]$ \\
$Q$ & Heat (power density) $\left[\mathrm{W} / \mathrm{m}^{3}\right]$ \\
$T$ & Temperature $[\mathrm{K}]$ \\
$V$ & Velocity $[\mathrm{m} / \mathrm{s}]$ \\
$V_{d}$ & Discharge voltage $[\mathrm{V}]$ \\
$a$ & Sound speed $[\mathrm{m} / \mathrm{s}]$ \\
$e$ & Elementary charge $[\mathrm{C}]$ \\
$j$ & Current density $\left[\mathrm{A} / \mathrm{m}^{2}\right]$ \\
$k$ & Boltzmann constant $[\mathrm{J} / \mathrm{K}]$ \\
$m$ & Particle mass $[\mathrm{kg}]$ \\
$\dot{m}$ & Mass flow rate $[\mathrm{mg} / \mathrm{s}]$ \\
$n$ & Number density $\left[\mathrm{m}^{-3}\right]$ \\
$p$ & Pressure $\left[\mathrm{N} / \mathrm{m}^{2}\right]$ \\
$s$ & Secondary electron emission coefficient \\
$t$ & Time $[\mathrm{s}]$ \\
$\beta$ & Ionization rate $\left[\mathrm{m}^{3} / \mathrm{s}\right]$ \\
$\mu$ & Mobility $\left[\mathrm{m}^{2} /(\mathrm{V} \mathrm{s})\right]$ \\
$\nu$ & Collision frequency $\left[\mathrm{s}^{-1}\right]$ \\
$\rho$ & Density $\left[\mathrm{kg} / \mathrm{m}^{3}\right]$ \\
$\sigma_{e n}$ & Electron-atom collision cross section $\left[\mathrm{m}^{2}\right]$ \\
$\left.\sigma_{\text {ion }}\right]$ & Electron impact ionization cross section $\left[\mathrm{m}^{2}\right]$ \\
$\phi$ & Potential $[\mathrm{V}]$ \\
\hline
\end{tabular}

\footnotetext{
*Graduate Student, Department of Aerospace Engineering, AIAA Student Member.

${ }^{\dagger}$ Assistant Research Scientist, Department of Aerospace Engineering, AIAA Senior Member.

${ }^{\ddagger}$ Doctor, Department of Aerospace Engineering, AIAA Member. Now with MIT Lincoln Laboratory, Lexington, MA 02420, USA

$\S$ Professor, Department of Aerospace Engineering, AIAA Associate Fellow.

ฯ Professor, Department of Aerospace Engineering, AIAA Associate Fellow.
} 


$\begin{array}{ll}\text { Subscript } & \\ a & \text { Neutral atom } \\ e & \text { Electron } \\ i & \text { Ion } \\ r & \text { Radial } \\ s & \text { Sheath } \\ z & \text { Axial }\end{array}$

\section{Introduction}

7 HERE is a growing interest in krypton as a propellant for Hall thrusters. Krypton offers several advantages over xenon as a propellant in electric propulsion devices. The most practical is the cost as krypton is more abundant and therefore less expensive than xenon. Krypton also offers a higher specific impulse, further lowering mission costs. On the other hand, krypton has been observed to have lower efficiencies stemming from a higher ionization potential, lower propellant utilization, and increased beam divergence. ${ }^{1,2}$ However $^{2}$ a couple of studies have shown that efficiencies comparable to xenon based thrusters can be achieved with krypton. ${ }^{2,3}$

Experimental work has been performed recently on the NASA-173Mv1 Hall thruster to characterize some of the differences between using xenon and krypton, particularly in trying to explain the performance issues of krypton. ${ }^{4-6}$ Internal plasma measurements were taken and analyzed. Krypton experiences a lower propellant utilization and a larger beam divergence, which appear to be the main contributors to its lower performance characteristics in general. Configuring the magnetic field topography to increase krypton propellant utilization, however, affects the beam divergence as well as other aspects of the plasma flow. A better understanding of the effects of the magnetic field topography as well as other thruster design inputs on the ionization and acceleration of the propellant gas may lead towards improving krypton efficiency in these thrusters.

A hydrodynamic model of the plasma flow within Hall thrusters is applied to the same thruster and operating conditions. This will complement the experimental data and provides additional analysis that is either difficult or unable to be performed physically. A description of the model is given below as well as comparisons to the experimental data and other observations.

\section{Method}

A hydrodynamic description of the quasi-neutral plasma flow within the thruster is used. ${ }^{7,8}$ The ions-only singly charged ions are considered for this model-are modeled using a finite-volume flux-splitting method on an axisymmetric Cartesian mesh. ${ }^{9}$ The governing ion equations can be written in conservative form as

$$
\begin{gathered}
\frac{\partial \rho}{\partial t}+\frac{\partial\left(\rho V_{z}\right)}{\partial z}+\frac{\partial\left(\rho V_{r}\right)}{\partial r}=\beta n_{a} \rho \\
\frac{\partial\left(\rho V_{z}\right)}{\partial t}+\frac{\partial\left(\rho a^{2}+\rho V_{z}^{2}\right)}{\partial z}+\frac{\partial\left(\rho V_{z} V_{r}\right)}{\partial r}=e n E_{z}+\beta n_{a} V_{a} \rho \\
\frac{\partial\left(\rho V_{r}\right)}{\partial t}+\frac{\partial\left(\rho V_{z} V_{r}\right)}{\partial z}+\frac{\partial\left(\rho a^{2}+\rho V_{r}^{2}\right)}{\partial r}=e n E_{r}
\end{gathered}
$$

The ions are assumed to be unmagnetized since the Larmor radius is much larger than the length of the channel. The ions are also assumed to be cold, eliminating the need for the ion energy equation as well as the pressure term in the momentum equations. The source terms on the right hand side of the above equations result from the effects of ionization and the electric field. The ionization rate is found from ${ }^{10}$

$$
\beta=\sigma_{\text {ion }} V_{t h}\left(1+2 \frac{k T_{e}}{e E_{\text {ion }}}\right) \exp \left(-\frac{e E_{\text {ion }}}{k T_{e}}\right)
$$

where $V_{t h}$ is the electron thermal velocity. The ionization cross section is dependent on electron energy, but for simplicity it is approximated here as a constant. ${ }^{11}$ The components of the electric field are calculated 
from the electron momentum equation,

$$
\nabla \cdot\left(\rho \vec{V}_{e} \vec{V}_{e}\right)=-\nabla p_{e}-e n\left(\vec{E}+\vec{V}_{e} \times \vec{B}\right)-\rho_{e} \nu_{e} \vec{V}_{e}
$$

The electrons are assumed to be inertia-less and mainly constrained along the magnetic field lines. For this model, a one-dimensional approximation of the magnetic field profile is used. The field lines lie along the radial direction and the axial direction is across field lines. The electrons are assumed to move freely along these magnetic field lines resulting in zero current and thermal equilibrium in the radial direction. The electron pressure term can be folded into the conservative side of the ion momentum equations, Eqs. (2) and (3), through a new effective sound speed defined by

$$
a^{*}=\sqrt{a^{2}+\frac{k T_{e}}{m_{i}}}
$$

The ion momentum equations then become

$$
\begin{gathered}
\frac{\partial\left(\rho V_{z}\right)}{\partial t}+\frac{\partial\left(\rho\left(V_{z}^{2}+a^{* 2}\right)\right)}{\partial z}+\frac{\partial\left(\rho V_{z} V_{r}\right)}{\partial r}=\frac{j_{e_{z}}}{\mu_{e}}+\beta n_{a} V_{a} \rho \\
\frac{\partial\left(\rho V_{r}\right)}{\partial t}+\frac{\partial\left(\rho V_{z} V_{r}\right)}{\partial z}+\frac{\partial\left(\rho\left(V_{r}^{2}+a^{* 2}\right)\right)}{\partial r}=0
\end{gathered}
$$

The electron mobility across field lines is dependent on the effective electron collision frequency which is a sum of the collision frequencies of electrons with the ions, neutral atoms, and the walls. The wall collision frequency is estimated as ${ }^{12}$

$$
\nu_{\mathrm{wall}}=\frac{V_{t h}}{h} \exp \left(-\frac{e \phi_{\mathrm{s}}}{k T_{e}}\right)
$$

where $h$ is the width of the channel. The potential drop across the wall sheath, $\phi_{\mathrm{s}}$, is described later along with the rest of the sheath properties. The system of equations is closed with the electron temperature calculated from an electron energy equation,

$$
\begin{gathered}
\frac{3}{2} \frac{\partial\left(j_{e} T_{e}\right)}{\partial z}=Q_{\text {joule }}-Q_{\text {ion }}-Q_{\text {wall }}-Q_{\mathrm{el}} \\
Q_{\text {joule }}=j_{e} E_{z} \\
Q_{\text {ion }}=e n n_{a} E_{\text {ion }} \beta \\
Q_{\text {wall }}=\nu_{\text {wall }} n e\left(2 k T_{e}+(1-s) \phi_{\mathrm{s}}\right) \\
Q_{\mathrm{el}}=3 \frac{m_{e}}{m_{i}} n \nu_{e a} k\left(T_{e}-T_{a}\right)
\end{gathered}
$$

that balances Joule heating with losses due to ionization, wall collisions, and elastic relaxation. ${ }^{12,13}$

The boundaries of the simulation are set at the sheath edge instead of the actual wall boundary since several of the assumptions in the governing equations break down within the sheath. Following previous work, the Bohm condition is not assumed a priori at the entrance of the sheath. ${ }^{12}$ Rather a calculated relation between the electric field and the ion velocity at the sheath edge, as shown in Fig. 1, is used. The electric field at the sheath edge is then used to calculate the density gradient through ${ }^{14}$

$$
\nabla n=-\frac{k T_{e}}{e n E}
$$

The potential drop across the sheath also depends on the ion velocity entering the sheath and the secondary electron emission. ${ }^{15}$

$$
\phi_{\mathrm{s}}=\frac{k T_{e}}{e} \ln \left(\frac{1-s}{V_{\mathrm{s}} \sqrt{2 \pi m_{e} /\left(k T_{e}\right)}}\right)
$$

The secondary electron emission coefficient is modeled after experimental observations. ${ }^{16}$

$$
s=0.54+\frac{1-0.54}{40} \frac{k T_{e}}{e}
$$


For the neutral atoms, a one-dimensional flow is considered. The density of the neutrals is also governed by a continuity equation similar to Eq. (1) except the source term is negative since ionization depletes the neutrals. For simplicity, the neutral axial velocity is kept constant.

For the governing ion equations, Eqs. (1), (7), and (8), a Roe solver is used to calculate the fluxes across each of the cell interfaces, which in turn are used to update the cell values at each time step. ${ }^{17}$ The mesh consists of $190 \times 127$ square cells $0.2 \mathrm{~mm}$ on a side. The simulation is iterated until it converges to a representative state. The simulation is run for the NASA-173Mv1 thruster using both xenon and krypton as the propellant. Both cases are run at the same power level of $500 \mathrm{~V}$ and $9.27 \mathrm{~A}$. Differences in the external applied conditions include the anode mass flow rate and the magnetic field configuration. ${ }^{4}$ Inherent differences between krypton and xenon include mass, ionization energy, and cross sections. These differences between the two cases are summed up in Table 1.

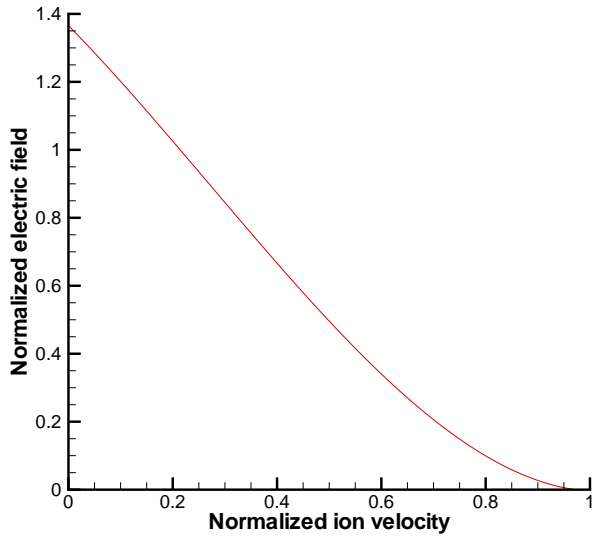

Figure 1. The calculated relation between the electric field, normalized by the electron temperature over the Debye length, and the ion velocity, normalized by the Bohm velocity, at the sheath edge.

\begin{tabular}{lcc}
\hline \hline & $\mathrm{Xe}$ & $\mathrm{Kr}$ \\
\hline$m_{i}[\mathrm{~kg}]$ & $2.18 \times 10^{-25}$ & $1.39 \times 10^{-25}$ \\
$E_{\text {ion }}[\mathrm{eV}]$ & 12.1 & 14.0 \\
$\sigma_{\text {ion }}\left[\mathrm{m}^{2}\right]$ & $3.6 \times 10^{-20}$ & $2.7 \times 10^{-20}$ \\
$\sigma_{\text {en }}\left[\mathrm{m}^{2}\right]$ & $35 \times 10^{-20}$ & $25 \times 10^{-20}$ \\
\hline$V_{d}[\mathrm{~V}]$ & 500 & 500 \\
$I_{d}[\mathrm{~A}]$ & 9.27 & 9.27 \\
$\dot{m}[\mathrm{mg} / \mathrm{s}]$ & 10.00 & 7.77 \\
\hline \hline
\end{tabular}

Table 1. Comparison between the xenon and krypton cases. The magnetic field profiles are another difference between the two.

The results of the simulation model are compared to experimental data obtained from tests run on the NASA-173Mv1 thruster in the Large Vacuum Test Facility (LVTF) at the Plasmadynamics and Electric Propulsion Laboratory (PEPL). ${ }^{4,6}$ The test chamber is a cylindrical tank $9 \mathrm{~m}$ in length and $6 \mathrm{~m}$ in diameter. The base pressure is $1.5 \times 10^{-7}$ torr and $3.3 \times 10^{-6}$ torr during operation. The High-Speed Axial Reciprocating Probe (HARP) system is used to probe inside of the thruster channel. ${ }^{18}$ A floating emissive probe and single Langmuir probe are attached to the HARP system and are swept into the thruster channel at high speeds such that the probes are kept in the channel for approximately $100 \mathrm{~ms}$. Full details of these experiments are discussed in previous work. ${ }^{4,6}$ The tests are run at the two operation points outlined in the bottom half of Table 1. The magnetic field configurations are not kept the same between the two cases, but are rather optimized for maximum efficiency at the same power level for both propellants.

\section{Results and Discussion}

The potential profiles along the channel centerline are shown in Fig. 2 for the experimental data and the model using the one dimensional approximation of the magnetic field. The model does a fairly good job of capturing the overall potential drop within the thruster for the two cases, roughly $175 \mathrm{~V}$ for krypton and $275 \mathrm{~V}$ for xenon. However, the model fails to accurately capture the steep profiles of the potential drops as well as the location of the start of the acceleration region. The experimental results show a well defined location of where the acceleration region begins and it is clear that for krypton it lies further downstream than xenon. The results of the model stretch the potential drop over a larger region within the thruster. There is no clear demarcation of the start of the acceleration zone, and the two propellants show little difference until a potential drop of more than $50 \mathrm{~V}$ has already occurred. Experimental results also show that in addition to starting further upstream, the acceleration zone for krypton is longer than that of xenon. The model does not extend past the exit plane of the thruster, but it can be inferred from the potential profiles within 
the thruster that, qualitatively, the model also reflects this observation. The longer acceleration region for krypton attributes in part to the greater beam divergence observed for krypton which in turn affects its overall performance.

The above points are made clearer if the axial electric fields are compared. Again, it is seen that the simulation results show a wider and less sharply defined acceleration zone than do the experimental results. The electric field begins to rise earlier upstream, while the peak is further downstream, past the exit plane. Comparing between the xenon and krypton again, the peak electric field is notably lower for krypton than for xenon in the simulation results, while experimentally the difference between the magnitudes of the peaks is not as significant. Overall, the model underpredicts the electric field in the acceleration region.

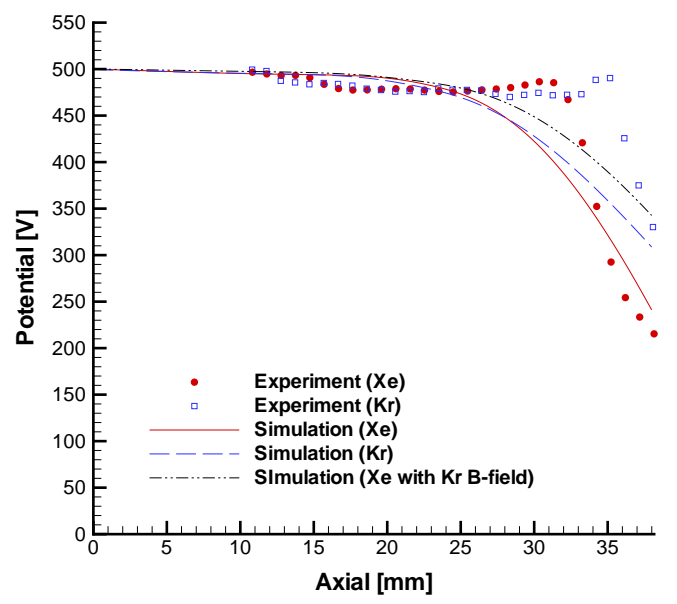

Figure 2. The channel centerline potential from anode to thruster exit plane for xenon and krypton.

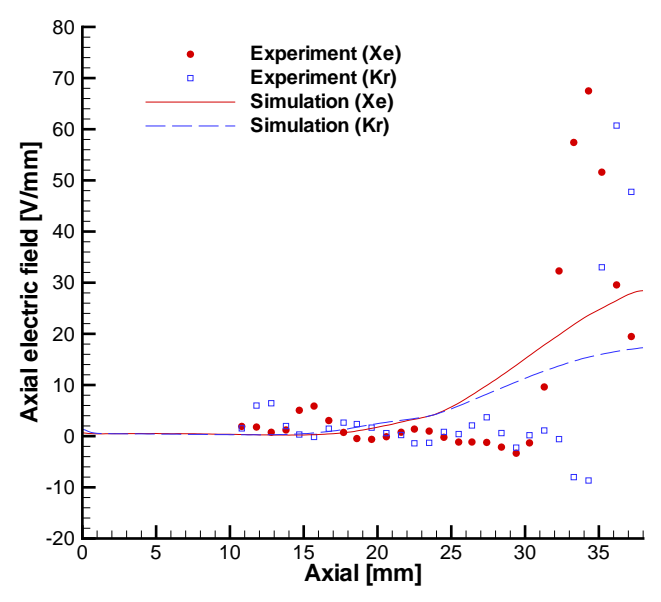

Figure 3. The axial electric field along the channel centerline.

The electron temperatures along the channel centerline are also compared. Figure 4 displays the results. Similar to the potential and electric field results, the model does not successfully capture the sharp rise, but rather smears the temperature increase. The peak temperatures calculated by the model are significantly lower than those measured. The model also does not capture the axial location of the peak accurately, especially for xenon. However, the model does predict the axial location of where the electron temperature begins to rise for the xenon case, and to a lesser extent, the krypton case.

The ionization rate along the channel centerline is displayed in Fig. 5 for xenon and krypton. The ionization rate is a function of electron temperature as seen in Eq. (4), and thus mirrors to some degree the electron temperature profiles for the two cases. The effects of the lower ionization potential and larger ionization cross section for xenon are clearly seen, though, especially in the downstream portions of the channel. The model does not capture a narrower ionization region, however, as the peak of the calculated ionization rate lies beyond the exit plane. This is due to the similar trends seen in the calculated electron temperature profile for both propellants.

The differences between the two cases arise either from the inherent differences between xenon and krypton or from the change in the mass flow rate or magnetic field profile. When xenon propellant is simulated using the magnetic field optimized for krypton, the resulting potential and electron temperature profiles, shown in Figs. 2 and 4, are more comparable to the krypton propellant case. The most direct effect the magnetic field has in the model is through the cross field electron mobility. The magnetic field configuration for xenon impedes electron mobility across field lines which increases the magnitude of the potential drop and conversely the magnetic field profile for krypton allows for a greater electron mobility and reduces the change in potential. Thus when xenon propellant is simulated with the magnetic field optimized for krypton, the resulting potential profile is more similar to that of the original krypton case. 


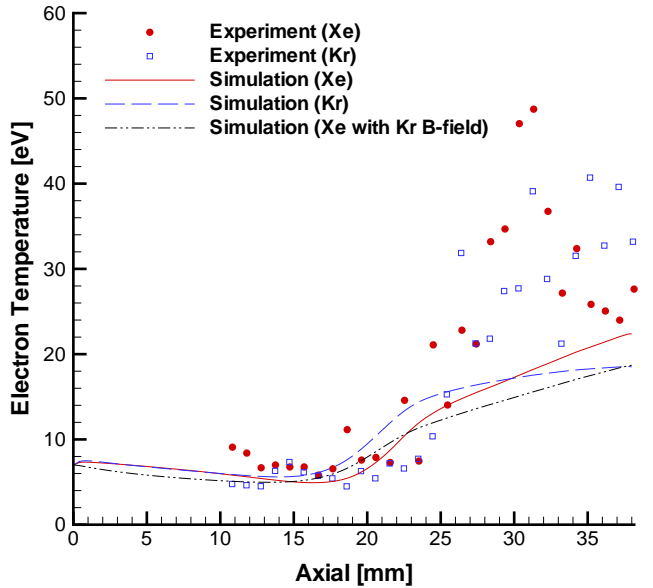

Figure 4. The electron temperature along the channel centerline.

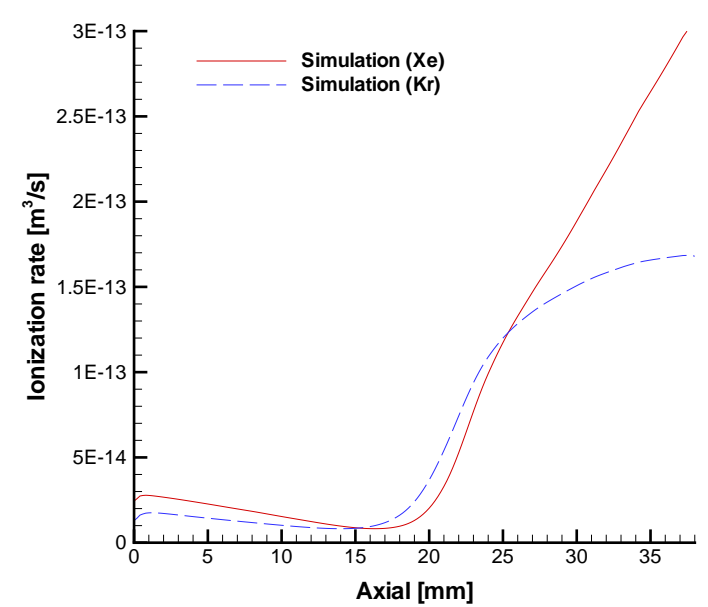

Figure 5. The calculated ionization rate along the channel centerline.

\section{Conclusions}

An axisymmetric hydrodynamic simulation using a one dimensional approximation of the magnetic field profile is used to model the plasma flow in the NASA-173Mv1 Hall thruster for both xenon and krypton propellants. Although quantitatively the results of the simulations underpredict the peak axial electric field and electron temperature, qualitatively, similar trends are observed when comparing the differences between the krypton and the xenon. Most notably, the acceleration zone for krypton is wider than that of xenon. With more of the acceleration occurring outside of the thruster, coupled with a magnetic field configuration that does not focus the plasma as well, it would be expected that krypton would have greater beam divergence than xenon, which is one of the reasons behind its reduction in performance. The magnetic field profile affects this most directly through the electron mobility. The differences between the two propellants in their ionization characteristics also account for part of the efficiency gap. This is most notably seen in the ionization rate, where xenon clearly ionizes more readily, even taking the differences of the electron temperature profiles into effect. Since propellant utilization is one of the main reasons behind krypton's poorer performance, it is important to investigate this area further to understand how to create improvements.

Future efforts will need to consider the effects of the two dimensional magnetic field profile, as it is one of the main differences between using the two propellants. Unfortunately, a magnetic profile optimized for one propellant may lead toward poor performance for another, thus can not be kept the same. Another improvement on the model would be to include the near field plume region, as a significant portion of the acceleration zone lies past the exit plane. Further investigation of the reasons behind krypton's lower performance may reveal areas for which future thruster designs can improve for krypton usage, thus taking advantage of its benefits, namely reduced cost and improved specific impulse.

\section{Acknowledgments}

The first author would like to thank the National Science Foundation for their support through the Graduate Research Fellowship Program.

\section{References}

${ }^{1}$ Marrese, C., Gallimore, A. D., Haas, J., Foster, J., King, B., Kim, S. W., , and Khartov, S., "An Investigation of Stationary Plasma Thruster Performance with Krypton Propellant," 31st AIAA/SAE/ASME/ASEE Joint Propulsion Conference and Exhibit, July 1995, AIAA 95-2932.

${ }^{2}$ Peterson, P. Y., Jacobson, D. T., Manzella, D. H., and John, J. W., "The Performance and Wear Characterization of a High-Power High-Isp NASA Hall Thruster," 41st AIAA/SAE/ASME/ASEE Joint Propulsion Conference and Exhibit, July 2005, AIAA 2005-4243. 
${ }^{3}$ Jacobson, D. T. and Manzella, D. H., "50 kW Class Krypton Hall Thruster Performance," 39th AIAA/SAE/ASME/ASEE Joint Propulsion Conference and Exhibit, July 2003, AIAA 2003-4550.

${ }^{4}$ Linnell, J. A. and Gallimore, A. D., "Internal plasma potential measurements of a Hall thruster using xenon and krypton propellant," Physics of Plasmas, Vol. 13, 2006, pp. 093502.

${ }^{5}$ Linnell, J. A. and Gallimore, A. D., "Krypton Performance Optimization in High-Voltage Hall Thrusters," Journal of Propulsion and Power, Vol. 22, No. 4, 2006, pp. 921-925.

${ }^{6}$ Linnell, J. A., An Evaluation of Krypton Propellant in Hall Thrusters, Ph.D. thesis, University of Michigan, 2007.

${ }^{7}$ Yim, J. T., Keidar, M., and Boyd, I. D., "A Hydrodynamic-Based Erosion Model for Hall Thrusters," 29th International Electric Propulsion Conference, Oct. 2005, IEPC-2005-013.

${ }^{8}$ Yim, J. T., Keidar, M., and Boyd, I. D., "An Investigation of Factors Involved in Hall Thruster Wall Erosion Modeling," 42nd AIAA/SAE/ASME/ASEE Joint Propulsion Conference and Exhibit, July 2006, AIAA 2006-4657.

${ }^{9}$ LeVeque, R. J., "Wave Propagation Algorithms for Hyperbolic Systems," Journal of Computational Physics, Vol. 131, No. 2, 1997, pp. 327-353.

${ }^{10}$ Ahedo, E., Martinez, P., and Martinez-Sanchez, M., "Steady and Linearly-Unsteady Analysis of a Hall Thruster with an Internal Sonic Point," 36th AIAA/SAE/ASME/ASEE Joint Propulsion Conference and Exhibit, July 2000, AIAA $2000-3655$.

${ }^{11}$ Sorokin, A. A., Shmaenok, L. A., Bobachev, S. V., Möbus, B., Richter, M., and Ulm, G., "Measurements of ElectronImpact Ionization Cross Sections of Argon, Krypton, and Xenon by Comparison with Photoionization," Physical Review A, Vol. 61, 2000, pp. 022723.

${ }^{12}$ Keidar, M., Boyd, I. D., and Beilis, I. I., "Plasma Flow and Plasma-Wall Transition in Hall Thruster Channel," Physics of Plasmas, Vol. 8, No. 12, Dec. 2001, pp. 5315-5322.

${ }^{13}$ Kroesen, G. M. W., Schram, D. C., Timmermans, C. J., and de Haas, J. C. M., "The Energy Balance of a Plasma in Partial Local Thermodynamic Equilibrium," IEEE Transactions on Plasma Science, Vol. 18, No. 6, Dec. 1990, pp. 985-990.

${ }^{14}$ Godyak, V. A., "Modified Bohm Criterion for a Collisional Plasma," Physics Letters, Vol. 89, No. 2, April 1982 , pp. 80-81.

${ }^{15}$ Hobbs, G. D. and Wesson, J. A., "Heat Flow Through a Langmuir Sheath in the Presence of Electron Emission," Plasma Physics, Vol. 9, 1967, pp. 85-87.

${ }^{16}$ Dunaevsky, A., Raitses, Y., and Fisch, N. J., "Secondary Electron Emission from Dielectric Materials of a Hall Thruster with Segmented Electrodes," Physics of Plasmas, Vol. 10, No. 6, June 2003, pp. 2574-2577.

${ }^{17}$ Roe, P. L., "Approximate Riemann Solvers, Parameter Vectors, and Difference Schemes," Journal of Computational Physics, Vol. 135, No. 2, 1997, pp. 250-258.

${ }^{18}$ Haas, J. M., Gallimore, A. D., McFall, K., and Spanjers, G., "Development of a High-Speed, Reciprocating Electrostatic Probe System for Hall Thruster Interrogation," Review of Scientific Instruments, Vol. 71, 2000, pp. 4131-4138. 